\title{
Social defeat stress promotes tumor growth and angiogenesis by upregulating vascular endothelial growth factor/extracellular signal-regulated kinase/matrix metalloproteinase signaling in a mouse model of lung carcinoma
}

\author{
XIAO WU ${ }^{1 *}$, BAO-JUN LIU ${ }^{1 *}$, SHUMENG JI ${ }^{2 *}$, JING-FENG WU ${ }^{1}$, CHANG-QING XU $^{3}$, YI-JIE DU ${ }^{1}$, \\ XIAO-FANG YOU ${ }^{4}$, BEI LI ${ }^{1}$, JING-JING LE ${ }^{1}$, HAI-LIN XU ${ }^{1}$, XIAO-HONG DUAN ${ }^{1}$ and JING-CHENG DONG ${ }^{1}$ \\ ${ }^{1}$ Department of Integrative Medicine, Huashan Hospital, Fudan University, Shanghai 200041; \\ ${ }^{2}$ Department of Dermatology, Xintai People's Hospital, Xintai, Shandong $271200 ;{ }^{3}$ Affiliated Hospital, School of Medicine, \\ Hangzhou Normal University, Hangzhou, Zhejiang 310015; ${ }^{4}$ Department of Childhood and Adolescence, \\ Key Laboratory of Public Health Safety, Ministry of Education, School of Public Health, \\ Fudan University, Shanghai 200032, P.R. China
}

Received April 17, 2014; Accepted February 11, 2015

DOI: $10.3892 / \mathrm{mmr} .2015 .3559$

\begin{abstract}
Numerous epidemiological and experimental animal studies have indicated that chronic psychological stress may promote tumor development. However, the underlying molecular mechanisms by which chronic stress promotes tumorigenesis remain to be fully elucidated and animal models have not yet been well established. In the present study, an established mouse model of repeated social defeat stress (RSDS), was generated and used to investigate the effect of stress on tumor growth and metastasis. C57BL/6 mice were exposed to RSDS for 10 days, followed by subcutaneousl inoculation with Lewis lung carcinoma cells for seven days. The tumor weight and volume as well as the number of the lung metastatic nodules were then determined. Vascular endothelial growth factor (VEGF) serum levels were measured using ELISAs. In addition, expression levels of VEGF receptor (VEGFR) and L1 cell adhesion molecule (L1CAM) messenger (m)RNA were confirmed using reverse transcription quantitative polymerase chain reaction. Furthermore, protein expression levels of phosphorlyated extracellular signal-regulated kinase (pERK), matrix metalloproteinase (MMP)-2 and MMP-9 were examined using western blot analysis. The results showed that RSDS significantly increased the weight and the volume of the primary tumor
\end{abstract}

Correspondence to: Professor Jing-Cheng Dong, Department of Integrative Medicine, Huashan Hospital, Fudan University, 12 Middle Urumqi Road, Shanghai 200041, P.R. China

E-mail: jcdong2004@126.com

*Contributed equally

Key words: repeated social defeat stress, angiogenesis, metastasis, vascular endothelial growth factor, matrix metalloproteinases as well as the number of the lung metastatic nodules. Serum VEGF levels were significantly higher in the tumor-stress group compared with those of the unstressed tumor mice. In addition, tumors in stressed animals demonstrated markedly enhanced expression of VEGFR-2 and L1CAM mRNA as well as pERK, MMP-2 and MMP-9 protein expression. In conclusion, these results suggested that RSDS contributed to lung cancer progression, angiogenesis and metastasis, which was partially associated with increased VEGF secretion and therefore the activation of the ERK signaling pathway, resulting in the induction of MMP-2 and MMP-9 protein expression.

\section{Introduction}

Lung cancer is the most common type of cancer and the most prevalent cause of cancer-associated mortality worldwide, as well as the leading cause of mortality in Chinese males (1). At present, there are $>150$ million lung cancer patients worldwide, with >one million novel cases diagnosed per year (2). Psychological distress, including depression, has previously been associated with increased lung cancer incidence and mortality (3). In addition, diminishing the effect of psychological stress through social support, including the presence of a social network or psychological intervention, was reported to increase the survival time and decrease the metastatic rate of cancer patients (4).

Numerous epidemiological studies have provided evidence that chronic psychological stress may have negative influences on the onset, progression and mortality rate of various types of cancer (4-6). A meta-analysis of 165 longitudinal studies revealed that psychosocial factors and stressful life experiences were associated with an increased incidence of cancer as well as decreased survival rates (6). Furthermore, psychosocial interventions have been shown to improve quality of life among cancer patients (7). However, there is only limited evidence to suggest that chronic stress enhances tumorigenesis 
in vivo, the molecular mechanisms of which remain to be elucidated (8).

Psychological factors have been thought to be associated with the downregulation of cellular immune responses, including the number and type of lymphocytes in circulation, proliferative responses in vitro as well as antibody levels post-immunization (9). These proposed mechanisms were suggested to be involved in cancer defense, as cellular immunity were reported to have an important role in the defense against cancer cells (10). Following initiation of tumor development, angiogenesis has a critical role in the growth and metastasis of tumors through the constitutive expression of several angiogenic genes, including vascular endothelial growth factor (VEGF) and matrix metalloproteinases (MMPs), particularly MMP-2 and MMP-9, which are involved in extracellular matrix degradation and are crucial for the endothelial cell migration, organization and hence, angiogenesis (11). In vitro studies have shown that tumor tissue stimulated the production of VEGF via $\beta$-adrenergic signaling; this process was blocked by the $\beta$-blocker propranolol (12). In addition, it has been demonstrated that stress hormones modulated the migration and invasion of cancer cells through stimulating the production of MMPs in tumor as well as stromal cells, and also served as chemoattractants for processes such as cell migration $(13,14)$. For example, norepinephrine (NE) and epinephrine (E) were found to significantly stimulate the production of MMP-2 and MMP-9 in ovarian cancer cells (15). MMPs were reported to facilitate tumor cell invasion and contribute to lung cancer progression; MMP-2 and MMP-9 were found to be differentially regulated in patient samples of lung tumor cells, although the physiological factors which modulate lung cancer MMP expression remain to be elucidated (16).

Stress is a complex process involving environmental and psychosocial factors, which are known to initiate cascade information processing in the peripheral as well as the central nervous system (17). Animal models which replicate the pathogenesis of human disease are essential for understanding the effects of stress on cancer, amongst other diseases, as well as for investigating potential therapeutic interventions for the effective prevention of tumor progression or further disease complications. In the present study, an established mouse model of repeated social defeat stress (RSDS), which was previously determined to mimic numerous symptoms of depression in humans (18-20), was used to investigate the effect of stress on the growth and metastasis of a Lewis lung carcinoma (LLC)-bearing tumor model in C57BL/6J mice in vivo.

\section{Materials and methods}

Animals. A total of 32 male C57BL/6J mice, aged six weeks, were purchased from Shanghai Laboratory Animal Center (Shanghai, China) and housed in the animal experiment center of the Fudan University (Shanghai, China). Male CD-1 mice, aged four months, were obtained from Vital River (Beijing, China) and housed in individual cages. The mice were housed at four per cage in an aseptic room at a constant temperature $\left(23^{\circ} \mathrm{C}\right)$ under a 12 -h light/dark cycle (light on from 7:00 AM to 7:00 PM) with ad libitum access to sterilized food and water. All animal experiments were approved by the Fudan University Animal Care and Use
Committee. Every effort was made to minimize the number of animals used and their suffering.

RSDS paradigm. The social defeat stress model was performed as previously described $(19,20)$. In brief, male C57BL/6J mice were used as experimental mice while resident mice were CD-1 retired breeders, selected for their attack latencies which are reliably $<30 \mathrm{sec}$. Every day, each experimental mouse was introduced into the home cage of a novel resident aggressor for $5 \mathrm{~min}$ and was physically defeated. Then, the resident home cage was divided into two halves using a perforated plexiglass partition to allow sensory contact but preventing further physical contact. Residents and intruders were maintained in sensory contact for $24 \mathrm{~h}$. Experimental mice were submitted to social defeat for 10 consecutive days. Control animals were housed in identically partitioned cages with a paired mouse from the same genotype and switched to opposite sides of the partition daily.

Social avoidance evaluation. Social avoidance evaluation was performed 11 days following the last social defeat using a video tracking system (EthovisionXT with Social Interaction Module; Noldus Information Technology, Wageningen, Netherlands) in a two-trial social interaction task. Testing was performed under red-light conditions in a room isolated from external sound sources. The social interaction test consisted of two separate trials: Trial 1 (no-target) and Trial 2 (target present). In Trial 1 (2.5 min), experimental mice explored an open-field arena containing an empty wire mesh cage on one edge of the arena. In Trial 2 (2.5 min), experimental mice were re-introduced to the arena, now with an unfamiliar CD-1 aggressor positioned in the mesh cage. Noldus video tracking system software was used to measure the time spent in the interaction zone surrounding the target box. Interaction ratios were calculated as the time spent in the interaction zone in the 'target present' condition as a percentage of the time spent in the zone in the 'no target' condition.

Spontaneous metastasis of lung cancer model. The LLC cell line was obtained from Experimental Center of Shanghai Chest Hospital (Shanghai, China) and maintained in RPMI-1640 medium (Sigma-Aldrich, St. Louis, MO, USA) containing 10\% fetal bovine serum (FBS; Gibco Life Technologies, Grand Island, NY, USA), $100 \mathrm{KU} / 1$ penicillin and $100 \mathrm{mg} / 1$ streptomycin (Beyotime Institute of Biotechnology, Jiangsu, China) at $37^{\circ} \mathrm{C}$ in humidified atmosphere of $5 \% \mathrm{CO}_{2} / 95 \%$ air with medium replacement every two days. Cells in the logarithmic growth phase were used for experiments in the present study. Following seven-day acclimatization, mice were randomly divided into four groups (eight mice per group) as follows: Control group (C), stress group (S), tumor group (T) and tumor-stress group (T-S). Mice in the $\mathrm{S}$ and T-S groups were exposed to RSDS for 10 days, as described above, while the mice in the $\mathrm{C}$ and $\mathrm{T}$ groups were exposed to control conditions. Subsequently, $1 \times 10^{6}$ LLC cells ( $0.1 \mathrm{ml}$ single-cell suspension) were inoculated subcutaneously into the right axilla of mice in the $\mathrm{T}$ and $\mathrm{T}-\mathrm{S}$ groups and the same volume of PBS $(0.1 \mathrm{ml})$ as a control was injected into the $\mathrm{C}$ and $\mathrm{S}$ groups. Following seven days, the subcutaneous tumors were measured using calipers thrice weekly and the tumor volume was calculated using 
A

Social interaction test

LLC cells inoculation

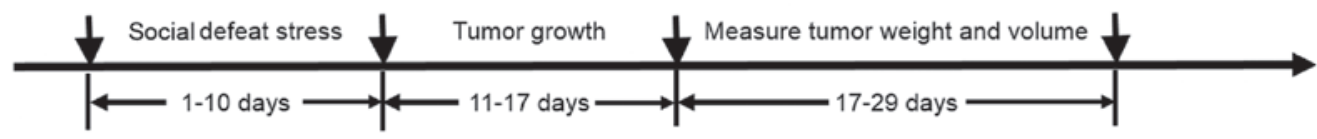

B

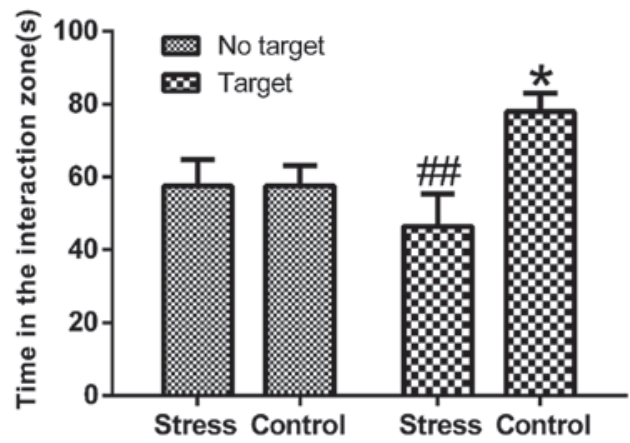

C

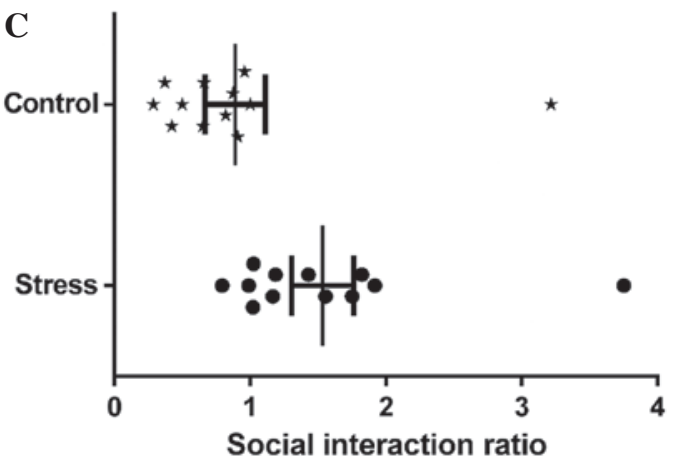

Figure 1. Repeated social defeat stress produces specific social avoidance of an aggressor animal. (A) Schematic depiction of the experimental procedures for the stress and tumor mice models. (B) Time spent in the interaction zone for control and defeated mice. (C) Interaction ratio, as determined by the proportion of time in presence vs. absence of a social target. Values are presented as the mean \pm standard error of the mean ( $\mathrm{n}=8)$. ${ }^{*} \mathrm{P}<0.05$ and ${ }^{\# \#} \mathrm{P}<0.01 \mathrm{vs}$. control and stress mice of the No target group, respectively. LLC, Lewis lung carcinoma.

the following formula: $\mathrm{V}=1 / 2\left(a b^{2}\right)$, where a represents the largest tumor diameter and b the smallest diameter. On day 29 the mice were sacrificed by cervical dislocation and tumors were harvested and weighed. Following blood collection, the lungs and tumors were rapidly excised and stored at $-80^{\circ} \mathrm{C}$ until assays were performed. The schematic diagram of the experimental procedures is shown in Fig. 1A.

Assessment of serum VEGF levels. Serum levels of VEGF were measured by ELISA. Blood was collected by intracardiac puncture and separated in a refrigerated centrifuge at $2,810 \mathrm{xg}$ for $10 \mathrm{~min}$ at $4^{\circ} \mathrm{C}$. Serum was separated from the clotted blood by centrifugation and analyzed for VEGF using the Mouse VEGF-A Platinum ELISA kit (eBiosciences, Inc., San Diego, CA, USA) according to the manufacturer's instructions.

Reverse transcription quantitative polymerase chain reaction $(R T-q P C R)$ analysis. Total RNA was extracted from the mouse tumors using TRIzol reagent (Invitrogen Life Technologies) according to the manufacturer's instructions. Samples containing $0.5 \mu \mathrm{g}$ total RNA were reverse-transcribed using oligo d(T) and a PrimeScript RT reagent kit (Takara Bio, Inc., Shiga, Japan). The resulting complementary DNA was then subjected to qPCR (IQ-5 System; Bio-Rad Laboratories, Inc., Hercules, CA, USA) according to the manufacturer's instructions. PCR amplification was conducted with the following cycling conditions: Enzyme activation at $95^{\circ} \mathrm{C}$ for $15 \mathrm{~min}$, followed by 40 cycles consisting of denaturation for $15 \mathrm{sec}$ at $95^{\circ} \mathrm{C}$, annealing for $30 \mathrm{sec}$ at $58-64^{\circ} \mathrm{C}$ depending on the primers, and elongation for $30 \mathrm{sec}$ at $72^{\circ} \mathrm{C}$. The primer sequences used were as follows: VEGF receptor 2 (VEGFR2) sense, 5'-GCTGTGAACGCTTGCCTTATGATG-3' and anti-sense, 5'-GCTCGCTGTGTGTTGCTCCTTC-3'; L1 cell adhesion molecule (L1CAM) sense, 5'-CTCCTCATCCTG CTCATCCTCTG-3' and anti-sense, 5'-GCCTTCTCTTCA TTGTCACTCTCC-3'; and GAPDH sense, 5'-AACTTGGCA TTGTGGAAGG-3' and anti-sense, 5'-GGATGCAGGGAT
GATGTTCT-3'. The primers were synthesized by Sangon Biotech Co., Ltd. (Shanghai, China) and the data were analyzed using the $2^{(-\Delta \Delta \mathrm{Ct})}$ method.

Western blot analysis. Mouse tumors were dissected, homogenized and resuspended in radioimmunoprecipitation assay lysis buffer. Lysates were kept on ice for $30 \mathrm{~min}$, vortexed, disrupted by 4 x 30-sec bursts of supersonication with a 550 Sonic Dismembrator (Thermo Fisher Scientific, Hampton, NH, USA) and then centrifuged at 10,800 x g for $20 \mathrm{~min}$ at $4^{\circ} \mathrm{C}$. Supernatants were collected and quantified for protein levels with a Bicinchoninic Acid Protein Assay kit (Beyotime Institute of Biotechnology) and a Synergy 2 MultiMode microplate reader $(562 \mathrm{~nm}$; BioTek Instruments, Inc., Winooski, VT, USA). In brief, supernatants were boiled in 2X SDS loading buffer (Beyotime Institute of Biotechnology), separated using 10\% SDS-PAGE and transferred to nitrocellulose membranes (Bio-Rad Laboratories, Inc.). The membrane was blocked with 5\% non-fat milk (Beyotime Institute of Biotechnology) for $1 \mathrm{~h}$ at room temperature and then incubated overnight at $4^{\circ} \mathrm{C}$ with the following primary antibodies: Mouse monoclonal anti-phosphorylated extracellular signal-regulated kinase (pERK; 1,000; sc-7383; Santa Cruz Biotechnology, Inc., Dallas, TX, USA), rabbit polyclonal anti-MMP-2 (1:2,000; ab124294; Abcam, Shanghai, China), rabbit monoclonal anti-MMP-9 (1:3,000; EP1255Y; Abcam) and anti- $\beta$-actin (1:1,000; AA132; Beyotime Institute of Biotechnology) antibodies. The blots were then incubated for $2 \mathrm{~h}$ at room temperature with goat anti-rabbit (A0208) and goat anti-mouse (A0216) horseradish peroxidase-conjugated goat secondary antibodies (1:3,000; Beyotime Institute of Biotechnology) in blocking buffer (Beyotime Institute of Biotechnology). Target proteins were detected using an enhanced chemiluminescence Plus Western Blotting Detection System (Amersham Pharmacia Biotech, Uppsala, Sweden) and images of the blots were captured using a molecular imager (GelDoc XR system; Bio-Rad Laboratories, Inc.). The images were analyzed using 

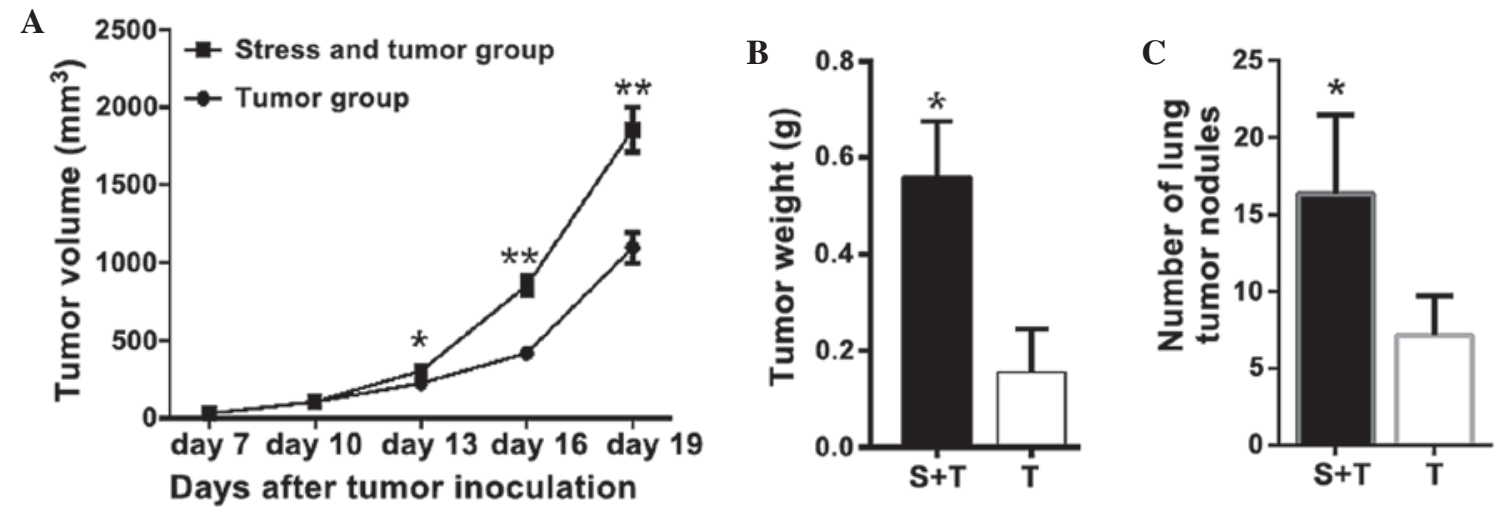

Figure 2. Repeated social defeat stress promotes Lewis lung carcinoma cell growth in vivo. (A) For 19 days following tumor inoculation, the tumor size was measured and calculated. On day 19 following tumor inoculation, mice were sacrificed and the tumors were harvested. (B) The tumor weight was calculated and $(\mathrm{C})$ the number of tumor nodules was counted in the lungs. Values are presented as the mean \pm standard error of the mean ( $=8$ per group). ${ }^{*}<0.05$ and ${ }^{* *} \mathrm{P}<0.01$ vs. T mice. T, tumor group; $\mathrm{S}+\mathrm{T}$, stress and tumor group.
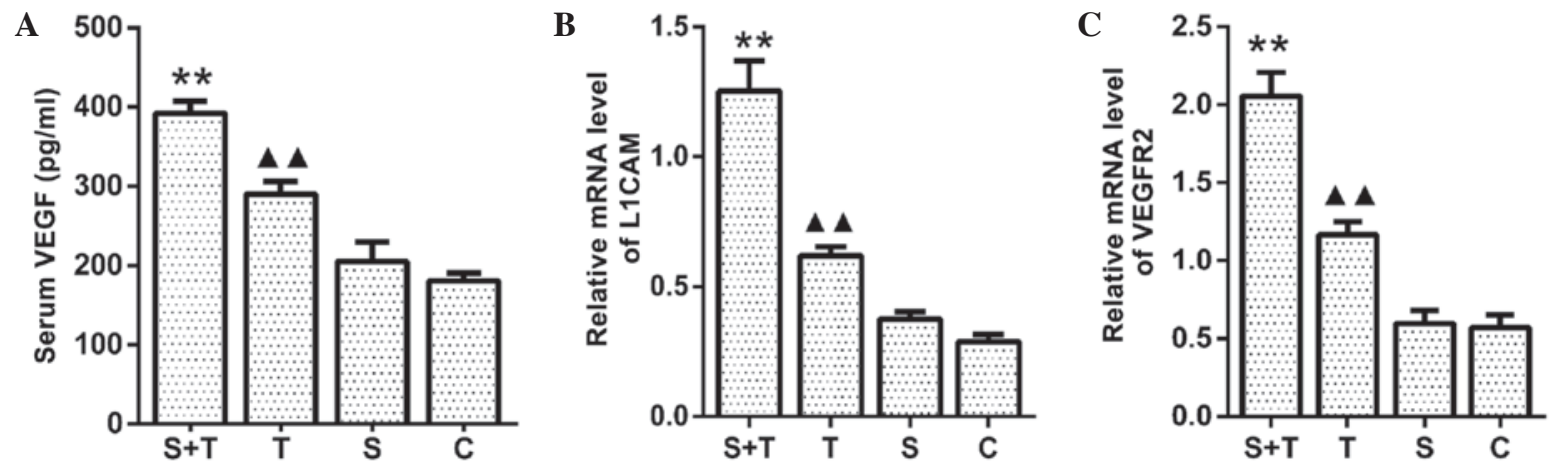

Figure 3. Repeated social defeat stress promotes Lewis lung carcinoma angiogenesis. (A) ELISA analyses of serum levels of VEGF. Reverse transcription quantitative polymerase chain reaction analysis of (B) L1CAM and (C) VEGFR2 mRNA expression levels in the lung tissue of C and S mice as well as the primary tumor of $\mathrm{T}$ and $\mathrm{T}+\mathrm{S}$ mice. Values are presented as the mean \pm standard error of the mean ( $\mathrm{n}=8$ per group). ${ }^{* *} \mathrm{P}<0.01 \mathrm{vs}$. $\mathrm{T}$ mice and $\boldsymbol{\wedge} \mathbf{\Delta}<0.01 \mathrm{vs}$. $\mathrm{C}$ mice. $\mathrm{T}$, tumor group; S+T, stress and tumor group; S, stress-only group; C, control group; VEGF, vascular endothelial growth factor; L1CAM, L1 cell adhesion molecule; VEGFR2, VEGF receptor type 2.

Quantity One image analysis software, version 4.2.1 (Bio-Rad Laboratories, Inc.). The optical densities of the target protein were normalized to that of $\beta$-actin. Western blot experiments were replicated three times.

Statistical analysis. Values are presented as the mean \pm standard error of the mean. Data analysis was performed using one-way analysis of variance or, for comparison of two groups, Student's $t$-test, conducted using SPSS, version 20.0 (IBM SPSS, Armonk, NY, USA). P $<0.05$ was considered to indicate a statistically significant difference between values.

\section{Results}

RSDS causes behavioral sensitization to stress. Mice were subjected to the ten-day social defeat procedure and then tested for social approach or avoidance behavior in a social interaction test. This was measured by comparing the time a mouse spent in an interaction zone with a social target to the time in that zone in the absence of a social target, as previously described (21). As shown in Fig. 1B and C, in the absence of an aggressor, the control and RSDS mice spent a comparable length of time in the interaction zone $(\mathrm{P}>0.05)$. By contrast, when an aggressor was introduced into the cage, control mice showed a dramatic increase in their interaction time, while chronically defeated mice showed a significant reduction in their interaction time $(\mathrm{P}<0.01)$ compared with that in the absence of an aggressor, which implied the successful establishment of the RSDS model.

RSDS promotes lung cancer progression. The effects of RSRS on lung cancer progression were investigated. Mice in the $\mathrm{T}$ and T-S groups were injected with LLC cells and the same volume of PBS was injected into the $\mathrm{C}$ and $\mathrm{S}$ groups as a control. No tumor formation was observed in mice in the $\mathrm{C}$ and $\mathrm{S}$ groups. As shown in Fig. 2A, tumors were first observed on day 7 and the volume of tumors increased until day 29, on which the mice were sacrificed. Tumor growth was significantly faster, with a markedly higher final tumor volume in the T-S group compared with that of the T group; the final tumor volume was $69.2 \%(\mathrm{P}<0.001)$ larger in the T-S group as compared with that of the $\mathrm{T}$ group. As shown in Fig. $2 \mathrm{~B}$, the mean weight of the tumors in the T-S mice was increased by $258 \%$ compared with that of the $\mathrm{T}$ mice. In addition, tumor nodules were also detected in the lungs of the $\mathrm{T}$ and $\mathrm{T}-\mathrm{S}$ groups when the mice were sacrificed 19 days following LLC cell injection. As shown in Fig. 2C, RSDS 

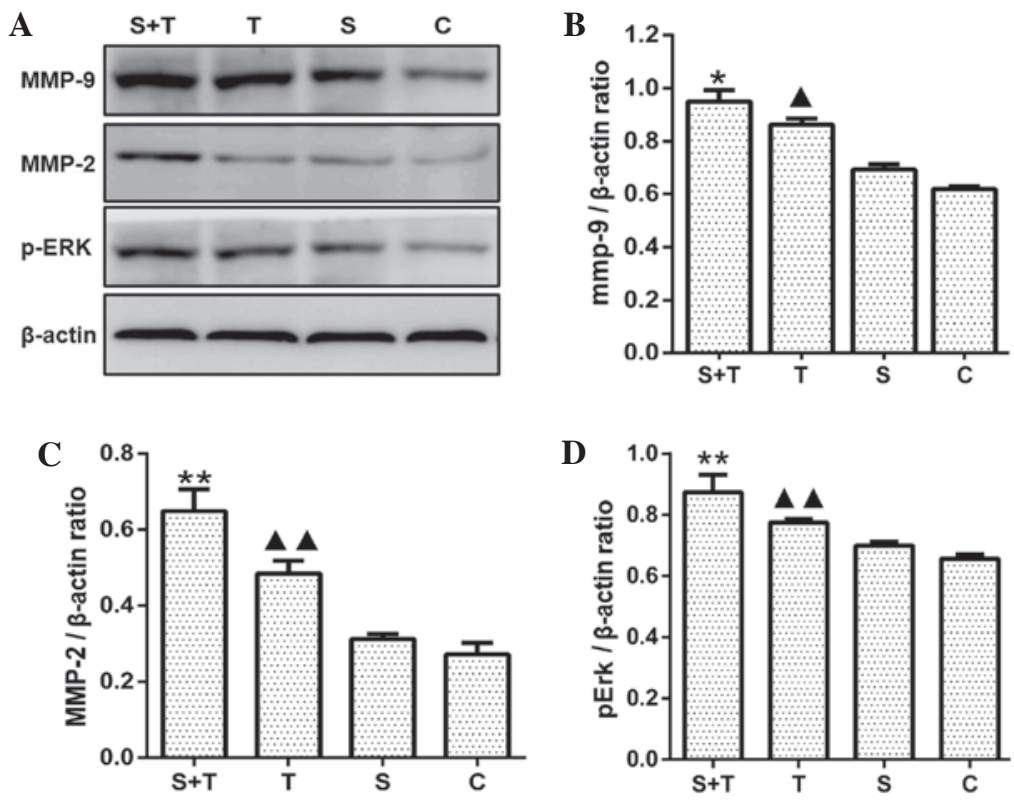

Figure 4. Repeated social defeat stress activates the ERK signaling pathway to induce MMP-2 and MMP-9 expression. (A) Western blot analysis of the protein expression of MMP-9, MMP-2 and pERK in the lung tissue of C and S mice as well as the primary tumor of T and T+S mice. Quantitative analysis of (B) MMP-9, (C) MMP-2 and (D) pERK expression. Absorbance ratios were normalized to that of $\beta$-actin. Values are presented as the mean \pm standard error of the mean ( $\mathrm{n}=4$ per group). ${ }^{*} \mathrm{P}<0.05$ and ${ }^{* *} \mathrm{P}<0.01$ vs. T mice, ${ }^{\wedge} \mathrm{P}<0.05$ and ${ }^{\wedge}{ }^{\wedge} \mathrm{P}<0.01$ vs. C mice. T, tumor group; S+T, stress and tumor group; $\mathrm{S}$, stress-only group; C, control group; MMP, matrix metalloproteinase; (p)ERK, (phosphorylated) extracellular signal-regulated kinase.

increased the number of tumor nodules on the lung by $128 \%$ $(\mathrm{P}<0.01)$, compared with that of the mice in the $\mathrm{T}$ group.

RSDS promotes the expression of VEGF, VEGFR2 and $L 1 C A M$. As previously described (10), the growth and metastasis of tumors is closely associated with angiogenesis. In the present study, it was investigated whether RSDS influenced the mRNA expression of VEGFR2 and L1CAM in the lung tissues and the serum levels of VEGF. As shown in Fig. 3A, serum VEGF levels were significantly higher in the T-S group compared with those of the unstressed $\mathrm{T}$ mice $(\mathrm{P}<0.05)$. Consistent with this, the mRNA expression of L1CAM and VEGFR2 in the lung tissue from the T-S mice was significantly increased compared with that in the T group $(\mathrm{P}<0.05)($ Fig. $3 \mathrm{~B}$ and $\mathrm{C}$ ). By contrast, no significant differences were observed in mRNA expression of VEGFR2 and L1CAM in the lung tissues or the serum levels of VEGF between the $\mathrm{S}$ and $\mathrm{C}$ groups $(\mathrm{P}<0.05)$. These results suggested that angiogenic processes mediated the effects of RSDS on tumor growth in LLC cell-bearing mice.

RSDS upregulates pERK, MMP-2 and MMP-9 protein expression. In order to verify whether upregulation of VEGF and VEGFR2 resulted in the activation of the gene transcription of downstream targets, western blot analysis was used to determine the expression levels of angiogenesis-associated proteins, including pERK, MMP-2 and MMP-9 in normal lung tissue from the $\mathrm{C}$ and $\mathrm{S}$ groups as well as tumor tissue from the $\mathrm{T}$ and T-S groups. The results showed that RSDS induced a significant increase in pERK, MMP-2 and MMP-9 protein expression levels compared with levels in the unstressed $\mathrm{T}$ group $(\mathrm{P}<0.01)$. No significant differences were noted in protein levels of pERK, MMP-2 and MMP-9 in normal lung tissues between the $\mathrm{S}$ and $\mathrm{C}$ groups ( $\mathrm{P}>0.05)$ (Fig. 4).

\section{Discussion}

Previous studies have revealed that psychological stress may be a potential modulator of cancer progression and this has become an important novel field of cancer research (22). Clinical and experimental animal studies indicated that stress, chronic depression and other psychological factors may influence the pathogenesis and progression of cancer. In the present study, an established RSDS mouse model, based on previous social defeat studies $(18,19)$, was used to explore the influence of stress on tumor growth and metastasis. The mechanisms by which chronic stress promoted tumorigenesis have previously been investigated (23). The results of the present study demonstrated that RSDS significantly increased the weight and volume of primary tumors as well as the number of the lung metastatic nodules. In addition, serum VEGF levels were significantly higher in the T-S group compared with those of the unstressed $\mathrm{T}$ mice. Furthermore, tumors in stressed animals showed markedly enhanced mRNA expression of VEGFR2 and L1CAM as well as protein levels of pERK, MMP-2 and MMP-9. These data suggested that RSDS contributed to lung cancer progression, angiogenesis and metastasis, which was partially associated with increased VEGF secretion and therefore activated the ERK signaling pathway, resulting in the induction of MMP-2 and MMP-9 expression.

Cancer metastasis, which is resistant to conventional therapies, is the primary cause of cancer-associated mortality (11). In addition, the result of cancer metastasis is dependent upon the tumor cells themselves as well as on the organ microenvironment (24). Therefore, treatments should be aimed at targeting the cancer cells as well as the host factors which contribute to the progressive growth and survival of metastatic cancer cells. Previous clinical and epidemiological studies have reported that psychosocial factors, including 
stress, chronic depression and lack of social support, may be risk factors for cancer progression $(6,15,25)$. However, there have been limited studies into the role of psychosocial factors in cancer initiation, although certain studies have reported that these factors may have a negative role in the progression of the cancer $(26,27)$. Studies have suggested that the chronicity of a negative effect sustains the activation of the pathways it mediates, which may be associated with cancer progression (28). Numerous studies have associated high levels of social support with improved clinical outcomes in cancer patients; in breast cancer patients, social support was associated with prolonged survival rates in multiple large-scale studies $(29,30)$, although negative findings were noted in certain studies (31). In view of the fact that there is still controversy over the role of psychosocial factors on carcinoma metastasis, further studies are required in order to verify this hypothesis and thereby provide more effective means for the potential treatment of cancer.

Based on previous findings, it was hypothesized that psychological factors reduce the body's immune system and have a positive role in the initiation and progression of cancer (19). Therefore, in the present study, an established RSDS mouse model, which was previously shown to mimic numerous symptoms of depression in humans $(18,19)$, was used to investigate the effect of stress on tumor growth and metastasis in a LLC-bearing tumor model.

Animal models which replicate the pathogenesis of human disease are essential for understanding the effects of stress on cancer, amongst other diseases (17). The consideration of chronic psychological stress due to socio-economic factors and disease-associated anxiety is an important, but overlooked aspect of cancer intervention that requires inclusion in preventative and therapeutic strategies for the successful treatment of lung cancer. The majority of previous studies have used various forms of chronic stress in order to replicate the behavioral adaptations associated with depression in animal models (32). These models include chronic unpredictable stress, restraint stress or foot-shock stress, which may be followed by behavioral measures of anhedonia (e.g., sucrose preference) or behavioral despair (e.g., forced swim test and tail suspension test) (33). However, all of these models are inconsistent with the etiology of the depression and cannot address the various validities which are required to produce an effective animal model of depression. The RSDS model used in the present study is performed on the basis of the etiology of the depression; it is comparable with the process of psychological factors acting on the human and better simulates the pathogenesis of human depression. In addition, another feature of this model is that social avoidance induced by 10 days of social defeat can be reserved through chronic, but not acute, anti-depressant treatments $(18,33)$. A previous study, which examined psychological distress and its association with the site of cancer reported that primary lung cancer was markedly associated with psychological distress in cancer patients (34). In addition, numerous studies have revealed that psychological distress was more prevalent in patients with lung cancer compared with other types of cancer (35). Therefore, in the present study, the RSDS model was combined with the spontaneous metastasis of lung carcinoma through inoculation of LLC cells in mice. This enabled the observation of the effect of psychological factors on the growth and metastasis of lung carcinoma.

Stress commonly occurs due to an imbalance between environmental requirements and an individual's abilities, and is often defined as the experience of a negative life event (36). Recently, studies have documented that stress may promote tumor incidence and progression (37). Metastasis is a complex process that requires the development of several hallmarks in order to occur, including angiogenesis, proliferation, invasion, embolization and evasion of immune system surveillance (38). Increasing evidence has suggested that the stress response may have roles is numerous aspects of this cascade, as cellular and molecular studies have associated stress with several processes known to be involved in cancer angiogenesis and metastasis (39). Angiogenesis involves the release of pro-angiogenic factors. VEGF is an angiogenic molecule which has important roles in embryogenesis, physiologic angiogenesis and the neovascularization of malignancies. Previous clinical studies have reported associations between increased levels of social support and lower VEGF expression in sera and tumor tissues (40). Furthermore, angiogenesis was found to be significantly upregulated in tumors in stressed mice compared with those in control mice; in addition, VEGF mRNA and protein levels were significantly elevated in tumor samples from mice exposed to daily stress (23). Consistent with these results, these present study demonstrated that serum VEGF levels were significantly increased in the T-S group compared with those in the unstressed $\mathrm{T}$ mice; in addition, the expression of VEGFR2 and L1CAM mRNA in lung tissue from the T-S group was significantly increased compared with that in the $\mathrm{T}$ group.

MMPs are a family of endoproteinases, which are essential for the degradation of the extracellular matrix and contribute to cancer initiation, growth, invasion and metastasis (41). The gelatinases A (MMP-2) and B (MMP-9) have been reported to be associated with an increased malignancy of tumor cells, as they are able to degrade type-IV collagen in the basement membrane (42), which was reported to be important for the invasive and metastatic potential in lung carcinoma (43). However, to date, the physiological factors involved in the modulation of MMP expression in lung cancer have remained to be elucidated. The results of the present study identified chronic stress as a novel regulator of MMP expression, which selectively upregulated MMP-9 and MMP-2 in the tumors of stressed animals. The ERK1/2 signaling pathways are important molecular pathways downstream of VEGF, which participate in regulating the expression of numerous genes (44). However, it has not yet been elucidated whether VEGF regulates the expression of MMP-2/9 through the ERK pathway. It was reported that estrogen increased the expression of VEGF, and thus activated ERK1/2 signaling to induce MMP-2/9 expression (45). In order to verify these findings in the present study, western blot analysis was used to detect the expression of pERK; the results showed that RSDS upregulated the expression of pERK in the tumor tissue of stressed mice. These data suggested that RSDS may contribute to lung cancer progression, angiogenesis and metastasis, which was found to be, in part, associated with increased VEGF secretion and there- 
fore activated the ERK pathway, resulting in the induction of MMP-2 and MMP-9 protein expression.

Future studies are required in order to further explore the specific molecular mechanism by which stress hormones modulate the interplay between tumor and stromal cells in the tumor microenvironment, resulting in the regulation of various signaling pathways associated with cancer progression. The elucidation of these pathways is therefore essential for the development of novel approaches to prevent and treat the deleterious effects of stress biology on cancer growth and metastasis.

In conclusion, the results of the present study expanded on the current understanding of the general pathways by which stress regulates cancer pathogenesis. These results have demonstrated that RSDS significantly promoted tumor growth, metastasis and angiogenesis, which was partially associated with increased VEGF secretion and the subsequent activation of the ERK signaling pathway, resulting in the induction of MMP-2 and MMP-9 protein expression. However, further studies are required in order to elucidate the psychoneuroimmunological details of the influence of chronic stress on lung cancer progression.

\section{Acknowledgements}

The present study was supported by grants from the National Natural Science Foundation of China (nos. 81173390 and 81102562), the National Basic Science Program of China (no. 2009CB523000) and the Chinese Ministry of Education Fund for Doctor Discipline Scientific Research (no. 20110071120072).

\section{References}

1. Zou XN, Lin DM, Wan X, et al: Histological subtypes of lung cancer in chinese males from 2000 to 2012. Biomed Environ Sci 27: 3-9, 2014.

2. de Mello RA, Costa BM, Reis RM and Hespanhol V: Insights into angiogenesis in non-small cell lung cancer: molecular mechanisms, polymorphic genes and targeted therapies. Recent Pat Anticancer Drug Discov 7: 118-131, 2012.

3. Al-Wadei HA, Plummer HR, Ullah MF, Unger B, Brody JR and Schuller HM: Social stress promotes and gamma-aminobutyric acid inhibits tumor growth in mouse models of non-small cell lung cancer. Cancer Prev Res (Phila) 5: 189-196, 2012.

4. Reiche EM, Nunes SO and Morimoto HK: Stress, depression, the immune system and cancer. Lancet Oncol 5: 617-625, 2004.

5. Antoni MH, Lutgendorf SK, Cole SW, et al: The influence of bio-behavioural factors on tumour biology: pathways and mechanisms. Nat Rev Cancer 6: 240-248, 2006.

6. Chida Y, Hamer M, Wardle J and Steptoe A: Do stress-related psychosocial factors contribute to cancer incidence and survival? Nat Clin Pract Oncol 5: 466-475, 2008.

7. Traeger L, Cannon S, Keating NL, et al: Race by sex differences in depression symptoms and psychosocial service use among non-Hispanic black and white patients with lung cancer. J Clin Oncol 32: 107-113, 2014.

8. Feng Z, Liu L, Zhang C, et al: Chronic restraint stress attenuates p53 function and promotes tumorigenesis. Proc Natl Acad Sci USA 109: 7013-7018, 2012.

9. Zorrilla EP, Luborsky L, McKay JR, et al: The relationship of depression and stressors to immunological assays: a meta-analytic review. Brain Behav Immun 15: 199-226, 2001.

10. Sahai E: Illuminating the metastatic process. Nat Rev Cancer 7: 737-749, 2007.

11. Fidler IJ, Kim SJ and Langley RR: The role of the organ microenvironment in the biology and therapy of cancer metastasis. J Cell Biochem 101: 927-936, 2007.
12. Liao X, Che X, Zhao W, Zhang D, Bi T and Wang G: The $\beta$-adrenoceptor antagonist, propranolol, induces human gastric cancer cell apoptosis and cell cycle arrest via inhibiting nuclear factor $\kappa \mathrm{B}$ signaling. Oncol Rep 24: 1669-1676, 2010.

13. Li S, Sun Y and Gao D: Role of the nervous system in cancer metastasis. Oncol Lett 5: 1101-1111, 2013.

14. Zou LB, Shi S, Zhang RJ, et al: Aquaporin-1 plays a crucial role in estrogen-induced tubulogenesis of vascular endothelial cells. J Clin Endocrinol Metab 98: E672-E682, 2013.

15. Spiegel D and Giese-Davis J: Depression and cancer: mechanisms and disease progression. Biol Psychiatry 54: 269-282, 2003.

16. Patil N, Ahmed Kabeer Rasheed S, Abba M, Hendrik Leupold J, Schwarzbach $\mathrm{M}$ and Allgayer $\mathrm{H}$ : A mechanistic study on the metastasis inducing function of FUS-CHOP fusion protein in liposarcoma. Int J Cancer 134: 2808-2819, 2014.

17. Moreno-Smith M, Lutgendorf SK and Sood AK: Impact of stress on cancer metastasis. Future Oncol 6: 1863-1881, 2010.

18. Berton O, McClung CA, Dileone RJ, et al: Essential role of BDNF in the mesolimbic dopamine pathway in social defeat stress. Science 311: 864-868, 2006.

19. Wu X, Wu J, Xia S, Li B and Dong J: Icaritin opposes the development of social aversion after defeat stress via increases of GR mRNA and BDNF mRNA in mice. Behav Brain Res 256: 602-608, 2013

20. Golden SA, Covington HR, Berton O and Russo SJ: A standardized protocol for repeated social defeat stress in mice. Nat Protoc 6: 1183-1191, 2011

21. Krishnan V, Han MH, Graham DL, et al: Molecular adaptations underlying susceptibility and resistance to social defeat in brain reward regions. Cell 131: 391-404, 2007.

22. Payne JK: State of the science: stress, inflammation, and cancer. Oncol Nurs Forum 41: 533-540, 2014.

23. Thaker PH, Han LY, Kamat AA, et al: Chronic stress promotes tumor growth and angiogenesis in a mouse model of ovarian carcinoma. Nat Med 12: 939-944, 2006.

24. Fidler IJ: The organ microenvironment and cancer metastasis. Differentiation 70: 498-505, 2002

25. Spiegel D: Health caring. Psychosocial support for patients with cancer. Cancer 74: 1453-1457, 1994.

26. Duijts SF, Zeegers MP and Borne BV: The association between stressful life events and breast cancer risk: a meta-analysis. Int J Cancer 107: 1023-1029, 2003.

27. Michael YL, Carlson NE, Chlebowski RT, et al: Influence of stressors on breast cancer incidence in the Women's Health Initiative. Health Psychol 28: 137-146, 2009.

28. Watson M, Haviland JS, Greer S, Davidson J and Bliss JM: Influence of psychological response on survival in breast cancer: a population-based cohort study. Lancet 354: 1331-1336, 1999.

29. Funch DP and Marshall J: The role of stress, social support and age in survival from breast cancer. J Psychosom Res 27: 77-83, 1983.

30. Giraldi T, Rodani MG, Cartei G and Grassi L: Psychosocial factors and breast cancer: a 6-year Italian follow-up study. Psychother Psychosom 66: 229-236, 1997.

31. Kroenke CH, Kubzansky LD, Schernhammer ES, Holmes MD and Kawachi I: Social networks, social support and survival after breast cancer diagnosis. J Clin Oncol 24: 1105-1111, 2006.

32. Mandelblatt JS, Hurria A, McDonald BC, et al: Cognitive effects of cancer and its treatments at the intersection of aging: what do we know; what do we need to know? Semin Oncol 40: 709-725, 2013.

33. Tsankova NM, Berton O, Renthal W, Kumar A, Neve RL and Nestler EJ: Sustained hippocampal chromatin regulation in a mouse model of depression and antidepressant action. Nat Neurosci 9: 519-525, 2006.

34. Dugan W, McDonald MV, Passik SD, Rosenfeld BD, Theobald D and Edgerton S: Use of the Zung Self-Rating Depression Scale in cancer patients: feasibility as a screening tool. Psychooncology 7 : 483-493, 1998

35. Zabora J, BrintzenhofeSzoc K, Curbow B, Hooker C and Piantadosi S: The prevalence of psychological distress by cancer site. Psychooncology 10: 19-28, 2001.

36. Strom TQ and Kosciulek J: Stress, appraisal and coping following mild traumatic brain injury. Brain Inj 21: 1137-1145, 2007.

37. Saul AN, Oberyszyn TM, Daugherty C, et al: Chronic stress and susceptibility to skin cancer. J Natl Cancer Inst 97: 1760-1767, 2005. 
38. Szlosarek P, Charles KA and Balkwill FR: Tumour necrosis factor-alpha as a tumour promoter. Eur J Cancer 42: 745-750, 2006.

39. Fang BA, Kovačević Ž, Park KC, et al: Molecular functions of the iron-regulated metastasis suppressor, NDRG1, and its potential as a molecular target for cancer therapy. Biochim Biophys Acta 1845: 1-19, 2014.

40. Schuller HM, Al-Wadei HA, Ullah MF and Plummer HR Regulation of pancreatic cancer by neuropsychological stress responses: a novel target for intervention. Carcinogenesis 33: 191-196, 2012.

41. Duffy MJ, Maguire TM, Hill A, McDermott E and O'Higgins N: Metalloproteinases: role in breast carcinogenesis, invasion and metastasis. Breast Cancer Res 2: 252-257, 2000.
42. Chambers AF and Matrisian LM: Changing views of the role of matrix metalloproteinases in metastasis. J Natl Cancer Inst 89: 1260-1270, 1997.

43. Shiraga M, Yano S, Yamamoto A, et al: Organ heterogeneity of host-derived matrix metalloproteinase expression and its involvement in multiple-organ metastasis by lung cancer cell lines. Cancer Res 62: 5967-5973, 2002.

44. WatanabeT,Takahashi A,SuzukiK,etal:Epithelial-mesenchymal transition in human gastric cancer cell lines induced by TNF- $\alpha$-inducing protein of Helicobacter pylori. Int J Cancer 134: 2373-2382, 2014.

45. Shan B,Li W, Yang SY and Li ZR: Estrogen up-regulates MMP2/9 expression in endometrial epithelial cell via VEGF-ERK1/2 pathway. Asian Pac J Trop Med 6: 826-830, 2013. 